\title{
Structural and electronic properties of pentacene-fullerene heterojunctions
}

\author{
Ingo Salzmann, ${ }^{1, a)}$ Steffen Duhm, ${ }^{1}$ Ricarda Opitz, ${ }^{1}$ Robert L. Johnson, ${ }^{2}$ Jürgen P. Rabe, ${ }^{1}$ \\ and Norbert Koch ${ }^{1, b)}$ \\ ${ }_{1}^{1}$ Institut für Physik, Humboldt-Universität zu Berlin, Newtonstraße 15, D-12489 Berlin, Germany \\ ${ }^{2}$ Institut für Experimentalphysik, Universität Hamburg, Luruper Chaussee 149, D-22761 Hamburg, Germany
}

(Received 7 September 2008; accepted 23 October 2008; published online 15 December 2008)

In this study the performance differences of layered and bulk-heterojunction based organic solar cells composed of the prototypical $p$ - and $n$-type organic semiconductors pentacene (PEN) and fullerene (C60) are correlated with the physical properties of the heterostructures. The electronic structure of layered and codeposited thin PEN and C60 films on the conducting polymer substrate poly(ethylenedioxythiophene):poly(styrenesulfonate) (PEDOT:PSS) was investigated with ultraviolet photoelectron spectroscopy. Layered structures of C60 on PEN precovered PEDOT:PSS exhibited an offset of the highest occupied molecular orbital (HOMO) levels of $1.45 \mathrm{eV}$. In contrast, codeposited films of PEN and C60 showed a reduced HOMO-level offset of $0.85 \mathrm{eV}$, which increased to $1.45 \mathrm{eV}$ by precoverage of the substrate with a thin PEN layer. In this case, the PEN-HOMO level was Fermi-level pinned at $0.35 \mathrm{eV}$ binding energy and charge transfer between PEN and PEDOT:PSS decreased the vacuum level by $0.75 \mathrm{eV}$. In addition, the morphology and crystal structure of the respective systems have been investigated by atomic force microscopy (AFM), x-ray diffraction (XRD) and Fourier-transform infrared spectroscopy, which indicated pronounced phase separation of PEN and C60 in the codeposited films. XRD revealed crystalline growth of PEN in all investigated cases forming crystallites that exceeded the nominal film thickness by an order of magnitude, whereas C60 was crystalline only if grown on the PEN precovered substrates. AFM investigations allowed to correlate morphology and structure revealing micro- and nanophase separation between PEN and C60. (C) 2008 American Institute of Physics. [DOI: $10.1063 / 1.3040003$ ]

\section{INTRODUCTION}

(Poly-)crystalline films of conjugated organic molecules are of interest for a large variety of applications in the field of organic electronics, including in particular light emitting diodes, thin film transistors, and photovoltaic cells (OPVCs). Significant efforts have been directed toward improving the understanding of organic/organic heterostructure interfaces, since numerous applications are based on the combination of different organic compounds. ${ }^{1-4}$ Concerning OPVCs, the combination of preferentially hole and electron conducting materials in a layered or a mixed-bulk morphology has been shown to be suitable for the fabrication of efficient solar cells. ${ }^{5,6}$

The material pair pentacene (PEN) and fullerene (C60) [chemical structure; see Figs. 1(a) and 1(b)] has been reported as efficient heterojunction in OPVCs based on layered thin organic films. ${ }^{7-9}$ Alternatively, OPVCs based on bulk heterojunctions formed via codeposition have been reported to show enhanced device performance compared to layered heterojunctions, like in case of phthalocyanines combined with 3,4,9,10-perylene tetracarboxylic bis-benzimidazole ${ }^{5}$ or C60, ${ }^{10-14}$ which could be explained through improved charge transport as well as increased exciton dissociation efficiency at the bulk heterojunction. ${ }^{6}$ However, no comprehensive study on the comparison of layered and bulk heterojunctions

\footnotetext{
a)Electronic mail: ingo.salzmann@physik.hu-berlin.de.

${ }^{b)}$ Electronic mail: nkoch@physik.hu-berlin.de.
}

for PEN and C60 exists, although it is well established that PEN exhibits phase separation in case of codeposition ${ }^{15,16}$ and that PEN based codeposited OPVCs show auspicious performance results. ${ }^{17}$

The present work was motivated by our experimental finding that bulk-heterojunction OPVCs based on PEN and C60 codeposited films $(\mathrm{PEN}+\mathrm{C} 60)$ show significantly lower efficiency compared to the respective layered structures (Fig. 2). This is surprising, since we would expect PEN and C60 to exhibit phase separation, therefore maximizing the common donor/acceptor interface and the energy levels to be suitable for efficient exciton dissociation. The aim of this comprehensive study is to identify the key parameters, which

(a)

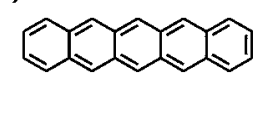

(c)

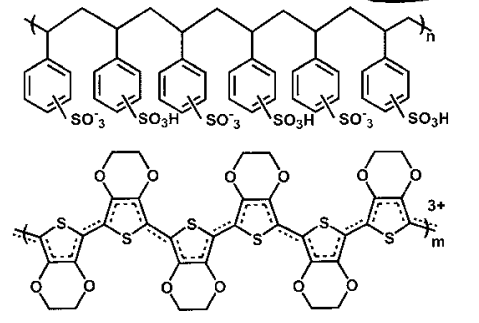

FIG. 1. The chemical structures of pentacene (a), C60 (b) and PEDOT:PSS (c). 


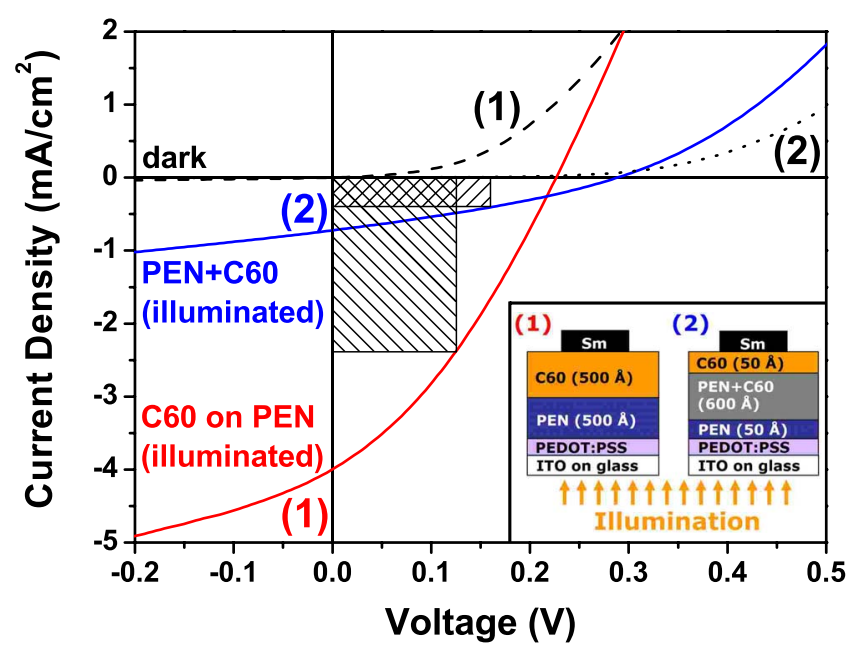

FIG. 2. (Color online) Representative current versus voltage characteristics of a layered C60 on PEN (curves labeled 1) and a bulk heterostructure based PEN + C60 OPVC (curves labeled 2) in dark (black) and under illumination (colored curves); the inset shows a cartoon of the OPVC structures. The shaded rectangles (areas: $P_{\max }=\max (J V)$, intersection with the $\mathrm{I}-\mathrm{V}$ curves at the maximum power point) in the power generating fourth quadrant indicate the fill factor. Device 2 exhibits a short circuit current density about six times lower than the layered structure.

lead to this unexpected result. We addressed this issue by (i) the thorough investigation of the electronic properties of layered and codeposited PEN+C60 heterojunctions on the application relevant conducting polymer electrode poly $(3,4-$ ethylenedioxythiophene)/poly(styrenesulfonate)

(PEDOT:PSS) [chemical structure; see Fig. 1(c)] using ultraviolet photoelectron spectroscopy (UPS). This is of highest interest, because the energy level alignment at interfaces plays a crucial role for the dissociation of photogenerated excitons as well as for the injection of charge carriers, which can act as bottleneck for organic device performance, ${ }^{4,18-22}$ (ii) We investigated the structural properties of layered and codeposited heterostructures using x-ray diffraction (XRD), since efficient bulk heterostructures require phase separation at the length scale of the exciton diffusion length, continuous paths of the pure materials to the electrodes as well as lowermost defect densities. ${ }^{5,13,18,23}$ (iii) We performed controlled heating experiments of PEN + C60 bulk heterostructures and investigated the impact on the structural order, since postfabrication annealing is a commonly applied tool to increase the device performance through structural rearrangement. ${ }^{5,24} \mathrm{Fi}$ nally (iv), we used atomic force microscopy (AFM) to study the morphology of the investigated heterosystems in conjunction with Fourier-transform infrared spectroscopy (FTIR) measurements to challenge our interpretations deduced from XRD and AFM.

\section{EXPERIMENTAL}

An aqueous dispersion of PEDOT:PSS [weight ratio of 1:20 (Baytron®P CH8000, H. C. Starck Gmbh \& Co. KG) $]^{25}$ was spin cast onto freshly cleaned and $\mathrm{UV} / \mathrm{O}_{3}$ treated $(30$ min) indium-tin oxide (ITO) coated glass substrates (8 $\times 8 \mathrm{~mm}^{2}$ size) under ambient conditions; the substrates were annealed at $200{ }^{\circ} \mathrm{C}$ for $5 \mathrm{~min}$ in ambient air and served as high work-function electrode. Photoemission experiments were performed at the FLIPPER II end-station at HASYLAB (Hamburg, Germany). ${ }^{26}$ The interconnected sample preparation chambers (base pressure $2 \times 10^{-9} \mathrm{mbar}$ ) and analysis chamber (base pressure $2 \times 10^{-10} \mathrm{mbar}$ ) allowed sample transfer without breaking the ultrahigh vacuum. Pentacene (Fluka, purum grade 99.9\%) and C60 (Sigma-Aldrich, purity 99.9\%) were used without further purification and were evaporated using resistively heated pinhole sources at evaporation rates of about $1 \AA /$ min; codeposition was performed via simultaneous evaporation from two spatially separated sources. The film mass thickness was monitored with a quartz crystal microbalance. Hence, the indicated values for coverages correspond to nominal film thicknesses. UPS spectra were recorded with a double-pass cylindrical mirror analyzer in off-normal geometry and with an acceptance angle of $24^{\circ}$ at an energy resolution of $200 \mathrm{meV}$ and a photon energy of $22 \mathrm{eV}$. The secondary electron cutoffs (SECOs) [for determination of the sample work function $(\phi)$ and the ionization energy (IE)] were measured with the sample biased at $-3.00 \mathrm{~V}$. The position of the substrate Fermi energy was determined using a freshly evaporated and sputtered $\mathrm{Au}-$ film. The error of all given values of binding energies (BEs) and SECO positions is estimated to $\pm 0.05 \mathrm{eV}$. All spectra shown were measured with the sample kept in dark and no spectral shift was observed when the samples were illuminated with white light from laboratory lamps thus ruling out surface photovoltaic effects during UPS measurements. $\mathrm{X}$-ray diffraction (XRD) measurements were performed at the beamline W1 at HASYLAB; the wavelength was set to $\lambda=1.1808 \AA$. The upper limit of instrumental broadening of the setup was estimated by the $2 \Theta$-width of the (111) reflection of a $\operatorname{Ag}(111)$ single crystal to $0.0382 \pm 0.0003^{\circ}$; line profiles were fitted using the pseudo-Voigt function. Additional structural investigations were performed on (100) $p$-doped silicon wafers with native oxide (Siegert Consulting, prime grade) as model substrates cut into $10 \times 10 \mathrm{~mm}$ coupons. The root mean square (RMS) roughness of the $\mathrm{SiO}_{2}$ substrates was determined to be below $2 \AA$ by AFM. The substrates were used as received; the cleanliness was confirmed prior to the film deposition by AFM investigations. Heating experiments were performed under $\mathrm{He}$ atmosphere; a constant temperature was held for $120 \mathrm{~min}$ at each step using a Lakeshore 330 temperature controller. All AFM investigations were done on Veeco Nanoscope III instruments in TappingMode ${ }^{\circ}$, recording topography and phase images. Current versus voltage curves ( $I-V$ curves) on OPVCs were recorded without breaking the vacuum immediately after device preparation using a Keithley 2400 General-Purpose SourceMeter (Keithley Instruments Inc.) under illumination through the glass substrate with a quartz halogen lamp (Solux, Light Bulbs Etc Inc., $12 \mathrm{~V}, 50 \mathrm{~W}, 4700 \mathrm{~K}$ with $10^{\circ}$ beam spread) providing $\sim 100 \mathrm{~mW} / \mathrm{cm}^{2}\left(P_{\text {inc }}\right)$ at the sample position. The top contacts for the $I-V$ measurements were vacuum sublimed samarium pads contacted with a flexible gold wire. FT-IR measurements (resolution $2.0 \mathrm{~cm}^{-1}$, nearnormal transmission geometry) were performed with a Bruker IFS-66v spectrometer. All preparation steps and measurements were performed at room temperature. 


\section{OPVC CHARACTERISTICS}

Layered and 1:1 codeposited OPVCs of PEN and C60 were investigated in vacuum by current versus voltage measurements. Representative $I-V$ curves are shown in Fig. 2; the inset depicts the respective device geometry and the nominal layer thicknesses. For the layered (C60/PEN) OPVC (blue curve in Fig. 2) we found an open circuit voltage $\left(V_{\mathrm{OC}}\right)$ of $0.24 \mathrm{~V}$, a short circuit current density $\left(J_{\mathrm{SC}}\right)$ of $4.15 \mathrm{~mA} / \mathrm{cm}^{2}$, and a fill factor $\mathrm{FF}=P_{\max } /\left(J_{\mathrm{SC}} V_{\mathrm{OC}}\right)$ of $33 \%$, where $P_{\max }=\max (J V)$ denotes the maximum power of the OPVC. For the bulk-heterojunction based OPVC (PEN + 660 on a thin PEN underlayer, red curve in Fig. 2) we found a slightly increased value for $V_{\mathrm{OC}}$ of $0.29 \mathrm{~V}$ and a significantly reduced value for $J_{\mathrm{SC}}$ of $0.73 \mathrm{~mA} / \mathrm{cm}^{2}$ (FF $=31 \%$ ). An evaluation of the power conversion efficiencies $\eta_{P}=J_{\mathrm{SC}} V_{\mathrm{OC}} \mathrm{FF} / P_{\mathrm{inc}}$ for the two device types (measured under identical experimental conditions) yielded an approximately six times lower value of $\eta_{P}$ for the bulkheterojunction devices. The result for the C60/PEN OPVC is comparable to published data ${ }^{8,27}$ taking into account the absence of a bathocuproine (BCP) exciton blocking layer in our OPVC. ${ }^{28}$ However, the bulk heterojunction OPVC showed reduced values for $J_{\mathrm{SC}}$ and $\eta_{P}$ by almost a factor of 6 . To investigate whether this result is caused by a modification of the electronic structures of PEN and C60 in case of codeposition we performed UPS on the pure, layered and codeposited samples of PEN and C60.

\section{ELECTRONIC STRUCTURE}

Figure 3 shows thickness dependent UPS spectra of layered and codeposited PEN and C60 films. From the SECO the sample work function can be derived and the emission onset of the highest occupied molecular orbital (HOMO) yields the hole injection barrier $\Delta_{H}$ (Ref. 19). The deposition of PEN with a nominal thickness $\left(\theta_{\mathrm{PEN}}\right)$ of $128 \AA$ on the PEDOT:PSS substrate $(\phi=5.30 \mathrm{eV})$ decreased the vacuum level by $0.75 \mathrm{eV}$ [see Fig. 3(a)]. This shift of the vacuum level can be attributed to an interfacial charge transfer since PEN is Fermi-level pinned on conducting polymer substrates with high work functions. ${ }^{29,30}$ The PEN spectrum in the valence electron region with the onset of the HOMO derived peak at $0.35 \mathrm{eV} \mathrm{BE}$ is consistent with earlier reported spectra of PEN on PEDODT:PSS. ${ }^{29}$ The subsequent deposition of C60 with a nominal thickness $\left(\theta_{\mathrm{C} 60}\right)$ of up to $16 \AA$ on top of the PEN underlayer did not further change the position of the SECO; i.e., C60 seems vacuum level aligned with PEN. However, further deposition of up to $64 \AA$ C60 increased the vacuum level by $0.15 \mathrm{eV}$. In the valence electron region the emission feature of the C60 HOMO level became apparent with the peak onset at $1.80 \mathrm{eV}$ BE. The PEN features were gradually attenuated by the C60 deposit and almost vanished at $\theta_{\mathrm{C} 60}=64 \AA$; the position of both the C60 and the PEN HOMO levels stayed essentially constant for all values of $\theta_{\mathrm{C} 60}$. Therefore, we conclude that $\mathrm{C} 60$ and PEN exhibit a HOMO level offset $\left(\Delta_{\text {HOMO }}\right)$ of $1.45 \mathrm{eV}$ in the layered arrangement C60/PEN on PEDOT:PSS. This is a relatively large value for $\Delta_{\text {Номо }}$, which is a crucial energetic parameter for efficient exciton dissociation in OPVCs accessible via
UPS. ${ }^{18}$ A recent UPS study of C60/PEN on polycrystalline $\mathrm{Au}$ substrates yielded similar results for the energy level offsets. $^{31}$

In the case of codeposition of PEN and C60 in a volume ratio of 1:1 on PEDOT:PSS without a PEN underlayer [see Fig. 3(b)] we found a small increase in the sample work function to $\phi=5.20 \mathrm{eV}$ for a nominal film thickness $\theta_{\mathrm{CO}}$ $\leq 16 \AA$, which can be deduced from the shift of the SECO to higher kinetic energy. This is the same trend as found in a previous study of pure C60 on PEDOT:PSS. ${ }^{32}$ Further PEN + C60 deposition of up to a nominal thickness $\left(\theta_{\mathrm{CO}}\right)$ of $64 \AA$ did not change $\phi$ (Ref. 33). In the valence electron region we found a superposition of the emission features that we attribute to PEN and C60. For $\theta_{\mathrm{CO}}$ up to $64 \AA$ the onset of the PEN HOMO emission was found at $0.30 \mathrm{eV} \mathrm{BE}$, which is essentially the same value as found for the pure PEN film (see above). However, the onset of the C60 HOMO emission was at $1.15 \mathrm{eV} \mathrm{BE}$ in case of codeposition, which leads to a reduced value for $\Delta_{\text {Номо }}$ of $0.85 \mathrm{eV}$. If we take into account the reported values for the PEN and C60 transport gap $\left(E_{g}^{T}\right)$ of $2.2 \mathrm{eV}$ (Ref. 20) and $2.6 \mathrm{eV},{ }^{34}$ respectively, we can estimate the offsets of the lowest unoccupied molecular orbital (LUMO) levels to $1.05 \mathrm{eV}$ for C60 on PEN and to $0.45 \mathrm{eV}$ for PEN+C60 (assuming identical polaron energies of the two systems).

One crucial prerequisite for efficient exciton dissociation at the donor/acceptor interface is that the exciton energy ( $E_{\text {ex }}$; obtained as transport gap minus exciton $\mathrm{BE}$ ) is larger than the offset in the HOMO of the donor and the LUMO of the acceptor. ${ }^{18}$ For C60 on PEN this offset is $1.15 \mathrm{eV}$, whereas for PEN +C60 on pristine PEDOT:PSS we derive an offset of $1.75 \mathrm{eV}$, which is already in the range of $E_{\mathrm{ex}}$ of PEN, as estimated from the optical gap $(1.90 \mathrm{eV}) .{ }^{15}$ Therefore the dissociation efficiency for excitons generated in PEN at a bulk-heterojunction interface to C60 grown on bare PEDOT:PSS may be far lower than at a layered heterojunction of C60 grown on a PEN underlayer. The change in the HOMO-LUMO level offset due to PEN precoverage is a consequence of the substrate work-function shift due to the Fermi-level pinning of PEN. Hence, it appears reasonable to precover the PEDOT:PSS substrate with PEN prior to PEN + 660 codeposition in order to achieve energetics suitable for efficient bulk-heterojunction OPVCs (as it was done for the bulk-heterojunction based devices shown in Fig. 2).

The results of UPS investigations of a codeposited PEN+C60 film on PEDOT:PSS precovered with $128 \AA$ PEN are shown in Fig. 3(c). The SECO position stayed constant upon PEN+C60 deposition after the initial decrease in $\phi$ by $0.80 \mathrm{eV}$ due to the PEN coverage of the substrate. In the valence electron region we found again a superposition of the PEN and C60 HOMO features with emission onsets at $0.35 \mathrm{eV} \mathrm{BE}$ and $1.80 \mathrm{eV} \mathrm{BE}$, respectively. The HOMO level positions stayed constant up to the final $\theta_{\mathrm{CO}}$ of $64 \AA$. These values are identical to the case of C60/PEN and the HOMO level offset for PEN+C60 structure on PEN was again 1.45 $\mathrm{eV}^{35}$

These findings demonstrate that the different OPVC performance of the two device structures shown in Fig. 2, i.e., the lower $J_{\mathrm{SC}}$ value in the codeposited case (on the PEN 
(a)

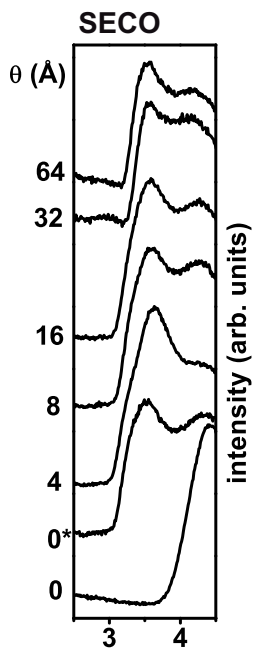

kinetic energy $(\mathrm{eV})$

(b)

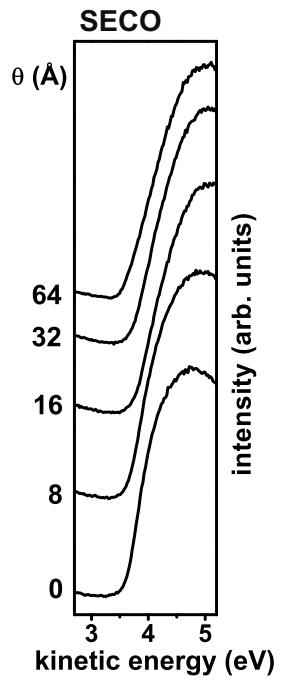

(c)

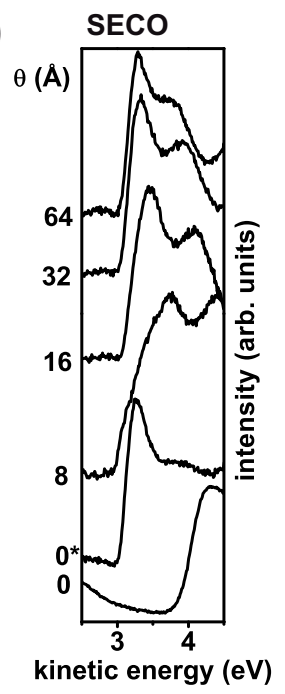

C60 / PEN / PEDOT:PSS
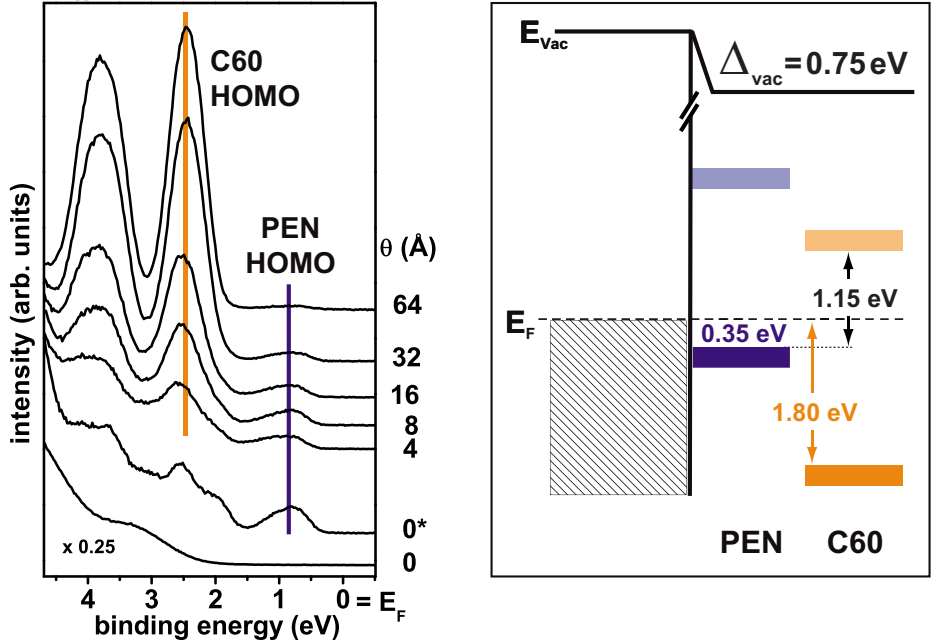

PEN+C60 / PEDOT:PSS
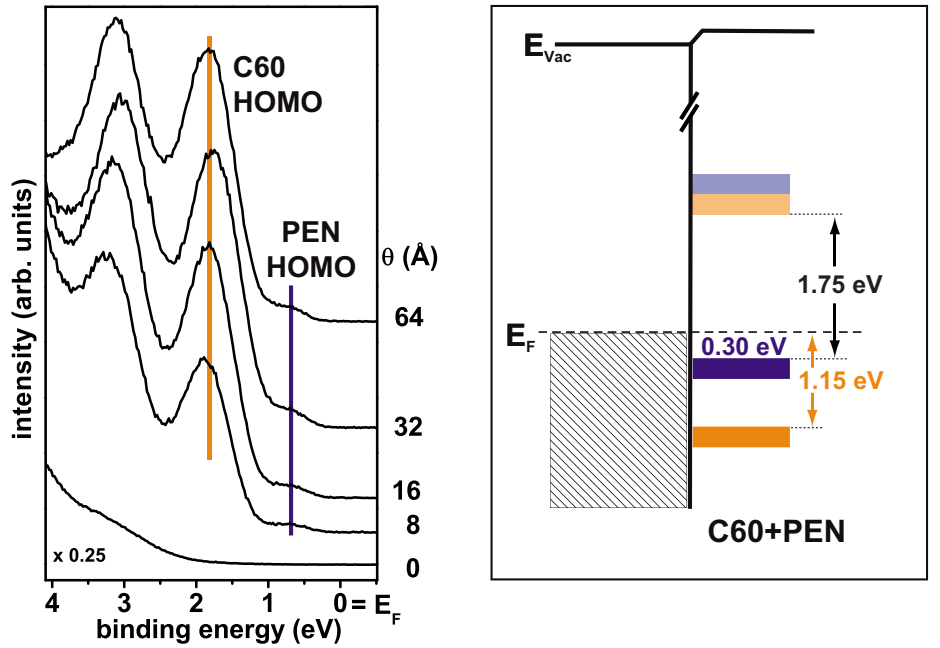

PEN+C60 / PEN / PEDOT:PSS

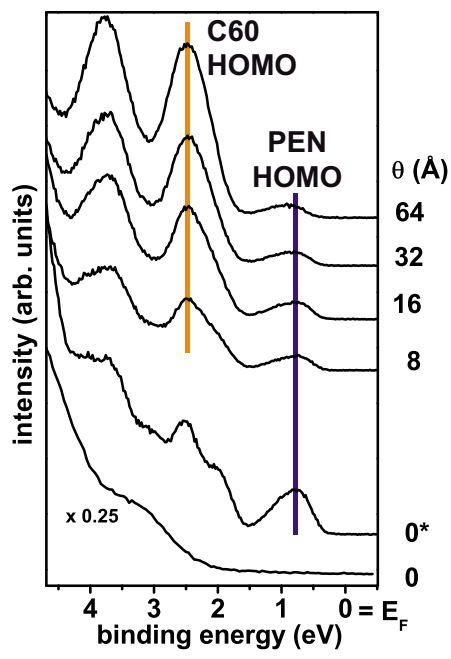

FIG. 3. (Color online) Thickness dependent UPS spectra of C60 on PEN precovered PEDOT:PPS (a), the codeposited PEN+C60 films on bare (b) and PEN precovered PEDOT:PSS (c) showing the SECO region (left panel), the valence orbital region (middle panel), and the respective schematic energy level diagram (right panel); vertical lines at the peak maxima in the valence electron region are guides for the eyes. $\theta$ denotes the nominal layer thickness, $E_{F}$ the Fermi energy, $\Delta_{\text {vac }}$ the shift of the vacuum level, and $0^{*}$ labels the spectrum of the 128 A PEN precoverage layers. In the schematic diagram dark and light colored rectangles depict HOMO and LUMO levels, respectively. The LUMO positions were estimated via the transport gaps. 

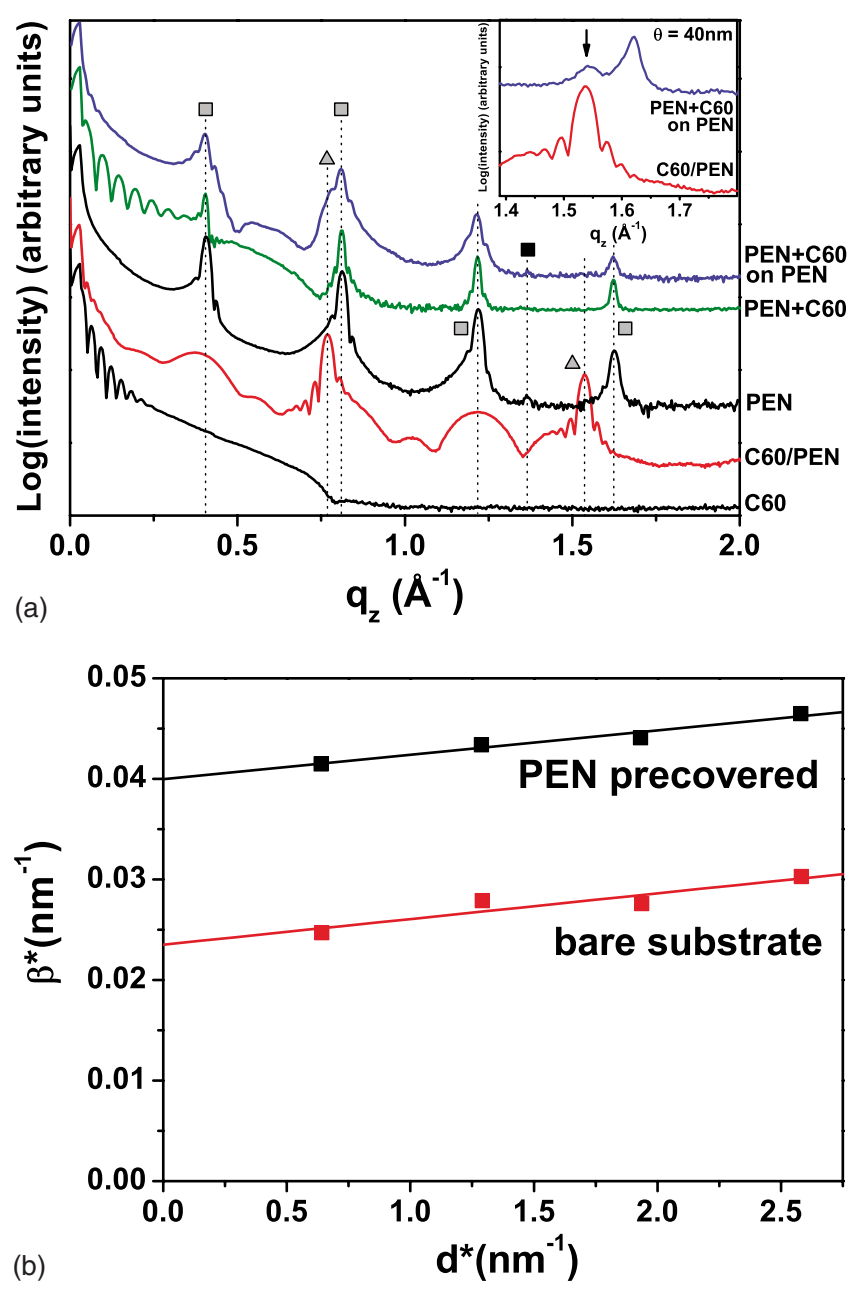

FIG. 4. (Color online) Specular x-ray diffraction results on films of nominally (from bottom to up): $20 \mathrm{~nm} \mathrm{C60,30} \mathrm{nm} \mathrm{C60} \mathrm{on} 3 \mathrm{~nm}$ PEN, $10 \mathrm{~nm}$ PEN, $20 \mathrm{~nm}$ PEN+C60 and $20 \mathrm{~nm}$ PEN+C60 on $3 \mathrm{~nm}$ PEN (a) and a WHA of the PEN reflections in the codeposited films (b). Peaks of the (001)-series from the PEN thin film phase are labeled with gray squares, the (002) and (004) reflections from C60 with triangles, and the black square denotes the (1-10) peak of a PEN bulk phase (Ref. 36). $q_{z}$ denotes the perpendicular momentum transfer with respect to the substrate plane, $\beta^{*}$ and $d^{*}$ denote the $2 \Theta$-integral peak breadth and the lattice spacing, respectively, expressed in reciprocal units. The inset in (a) shows a zoomed representation around the (004) peak of the C60 film on the PEN underlayer compared to the spectrum of a PEN+C60 film (nominal thickness $40 \mathrm{~nm}$ ) on PEN.

buffer layer), cannot be explained by differences of the respective PEN and C60 heterojunction energy levels. Therefore structural reasons may account for the different performance of the PEN+C60/PEN bulk heterojunction in comparison with the layered C60/PEN structure. This question was addressed via x-ray diffraction experiments on $\mathrm{SiO}_{2}$ as model substrate.

\section{STRUCTURAL INVESTIGATIONS}

We performed specular $\mathrm{x}$-ray diffraction investigations on pure $\operatorname{C60}\left(\theta_{\mathrm{C} 60}=20 \mathrm{~nm}\right)$ and $\operatorname{PEN}\left(\theta_{\mathrm{PEN}}=10 \mathrm{~nm}\right)$ films, on a C60 film $(\theta=30 \mathrm{~nm})$ on a thin PEN underlayer $\left(\theta_{\mathrm{PEN}}\right.$ $=3 \mathrm{~nm}$ ) as well as on codeposited PEN+C60 (total $\theta_{\mathrm{CO}}$ $=20 \mathrm{~nm}$ ) films on the bare and the PEN precovered substrate, the results are shown in Fig. 4(a). The pure C60 film showed pronounced thickness oscillations (Kiessig fringes ${ }^{37}$ ) corresponding to a layer thickness of $21 \pm 1 \mathrm{~nm}$ which stem from a smooth film of a thickness almost equal to the nominal film thickness. No Bragg peak could be observed thus indicating amorphous growth of $\mathrm{C} 60$ on bare $\mathrm{SiO}_{2}$. In contrast, on the approximately two monolayer thick PEN underlayer [broad PEN features marked with shaded squares in Fig. 4(a)] the C60 film exhibited the (002) and (004) peaks of hexagonally close packed $\mathrm{C} 60,{ }^{38}$ i.e., crystalline C60 through an ordering effect by the PEN underlayer growing in a $c^{*}$ axis orientation. ${ }^{39}$ We explain this effect by the characteristic distance of neighboring identical molecules in the (001) plane of the pentacene thin film phase ${ }^{40,41}(0.9637 \mathrm{~nm})$, which leads to a varying surface potential in the periodicity of the lattice constant $a$ of the hexagonal C60 polymorph $(1.0009 \mathrm{~nm})$ that initiates crystalline C60 growth in the specific polymorph, providing strong indications for heteroepitaxy of C60 and PEN. The intensity of the Kiessig fringes (corresponding to a film thickness of $33 \pm 1 \mathrm{~nm}$ ) is dramatically reduced in case of C60 on PEN, however, the thickness oscillations nicely correlate to the total nominal film thickness. From the spacing of the Laue oscillations ${ }^{42-44}$ in the vicinity of the first three C60 peaks [see inset of Fig. 4(a)] the coherent film thickness can be derived to $24 \pm 1 \mathrm{~nm}$, which indicates coherent out-of-plane order throughout almost the complete C60 film. The growth of PEN in the thin film phase (001-texture) on our $\mathrm{SiO}_{2}$ substrate is demonstrated for the pure PEN film in Fig. 4(a).

In the PEN+C60 film on bare $\mathrm{SiO}_{2}$ we found thickness oscillations corresponding to a smooth film of $13 \pm 1 \mathrm{~nm}$ and a series of pronounced Bragg peaks that correspond to the lattice spacing of the $00 l$-series of the PEN thin film phase, though no indication of crystalline C60 could be observed. However, for the PEN precovered film the (002) reflection of C60 can clearly be observed as shoulder of the PEN (001) peak; at a nominal film thickness of $\theta=40 \mathrm{~nm}$ even the (004) reflection can be found sufficiently separated from the PEN (004) peak [see inset of Fig. 4(a)]. This allowed to determine the out-of-plane crystalline coherence length of the crystalline C60 portion to $17 \pm 2 \mathrm{~nm}$ (estimated by the Scherrer formula ${ }^{45}$ ), i.e., almost half of the nominal film thickness.

The microstructure of the PEN portion of the codeposited film is an application relevant parameter, since defects or grain boundaries are limiting factors of device performance. ${ }^{46}$ The investigation of the microstructure was performed applying a Williamson-Hall analysis (WHA); ${ }^{16,47,48}$ the results are shown in Fig. 4(b). Herein, the total integral breadth $\left(\beta_{2 \Theta}\right)$ defined as the ratio of peak area and height is attributed to both diffraction order-independent broadening $\left(\beta_{S}\right)$ due to the average finite size of the crystallites and order dependent broadening $\left(\beta_{D}\right)$ due to strain fields introduced by dislocations (after the correction of the instrumental broadening). With WHA $\beta_{S}$ can thus be separated from $\beta_{D}$ by a plot of $\beta_{2 \Theta}^{*}=\beta_{S}^{*}+\beta_{D}^{*}=1 /\langle L\rangle_{V}+2 e d^{*}$, where $\langle L\rangle_{V}$ denotes the vertical volume-weighted average thickness of the crystallites and $e$ denotes the maximum (upper limit) strain that is proportional to the crystal lattice distortion; ${ }^{49-51}$ parameters expressed in reciprocal units are marked with $\left({ }^{*}\right)$ (Ref. 52). From WHA we found values of $42 \pm 2.5 \mathrm{~nm}$ and 


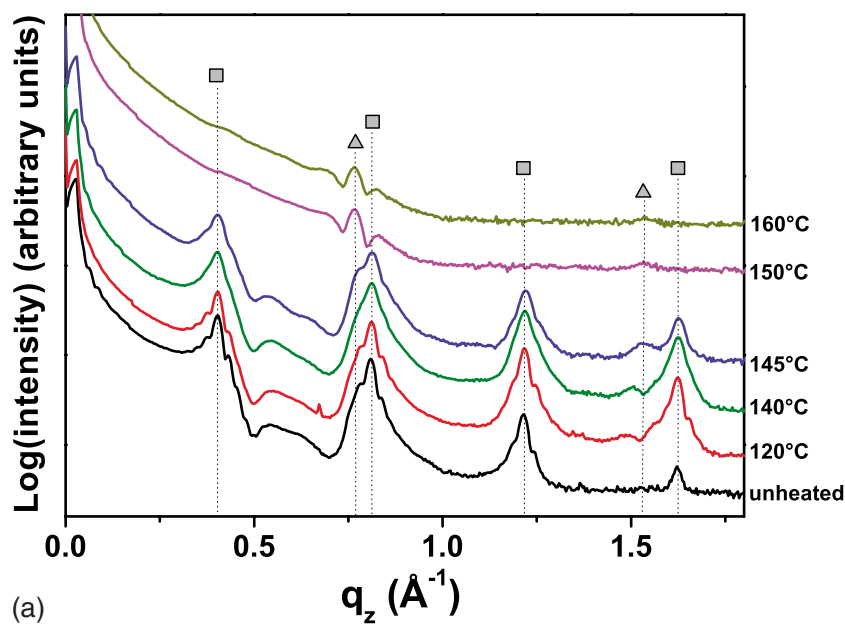

(a)

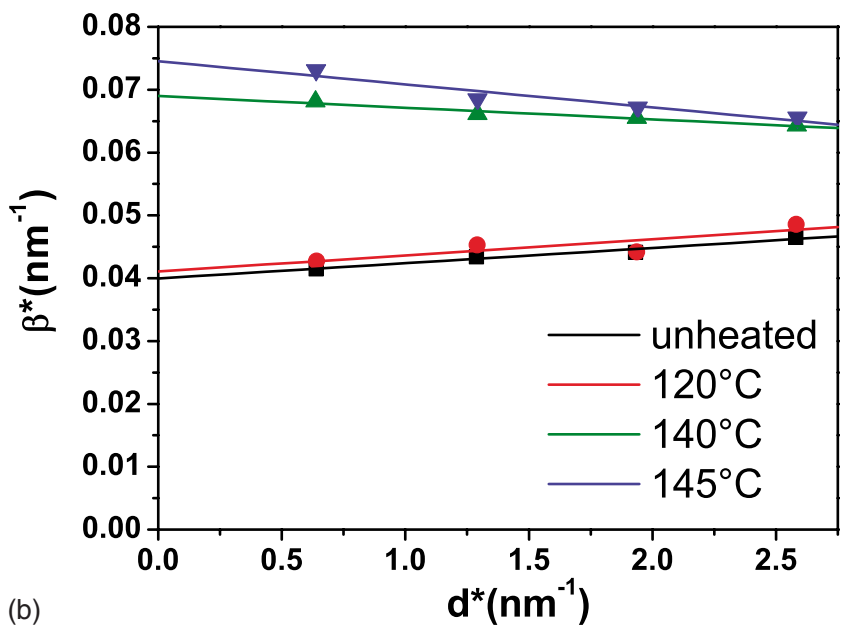

FIG. 5. (Color online) Specular x-ray diffraction measurements on annealed PEN + C60 structures $\left(\theta_{\mathrm{CO}}=20 \mathrm{~nm}\right)$ on PEN precovered $\mathrm{SiO}_{2}$ substrates (a) and the corresponding WHA (b). Peaks in (a) of the PEN thin film phase $00 l$-series are labeled with gray squares, the (002) and (004) reflections from C60 with triangles.

$25 \pm 0.5 \mathrm{~nm}$ for $\langle L\rangle_{V}$ of the PEN $+\mathrm{C} 60$ films on the bare and PEN precovered substrate, respectively. The microstrain was in both cases identical with $e=0.0025 \pm 0.0005$. Interestingly, this value is the same (within the error margin) as the value found for a pure PEN film of $\theta=30 \mathrm{~nm}(e$ $=0.0018 \pm 0.0005)$ in a previous study. ${ }^{16}$ An analogous analysis was done for the film of $\theta=40 \mathrm{~nm}$ and yielded values of $\langle L\rangle_{V}=87 \pm 12 \mathrm{~nm}(e=0.0038 \pm 0.0008)$ and $\langle L\rangle_{V}$ $=45 \pm 1 \mathrm{~nm}(e=0.0015 \pm 0.0003)$ on the bare and PEN precovered substrate, respectively.

In summary, our XRD results indicate that (i) PEN + C60 films exhibit phase separation, (ii) PEN grows in the thin film phase polymorph and forms islands with a height exceeding the nominal PEN mass thickness (i.e., $\left.\theta_{\mathrm{CO}} / 2\right)$ in the codeposited films by factors of 4 and 2 for the bare and the PEN precovered substrate, respectively, (iii) the PEN islands in the PEN+C60 films exhibit structural order comparable to pure PEN films, and (iv) PEN precoverage leads to an on ordering effect on $\mathrm{C} 60$ in the PEN+C60 film, as was also found for the layered structure of C60 on PEN.

It has been reported that postfabrication annealing of films of OPVC material pairs ${ }^{5,53}$ and pure C60 layers ${ }^{24}$ was

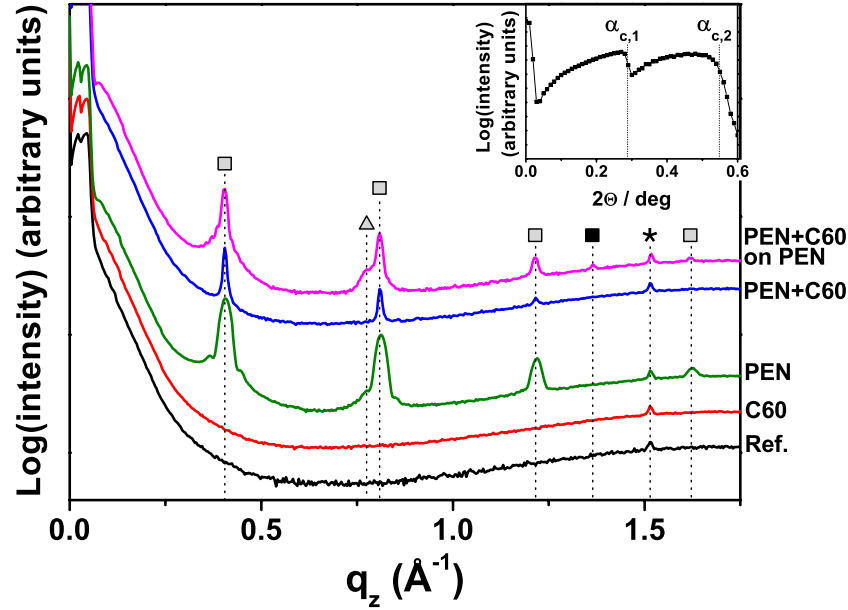

FIG. 6. (Color online) Specular x-ray diffraction on PEDOT:PSS coated ITO (from bottom to up): Reference PEDOT:PSS (CH8000) on ITO, $20 \mathrm{~nm}$ C60, $20 \mathrm{~nm}$ PEN, $40 \mathrm{~nm}$ PEN+C60, $40 \mathrm{~nm}$ PEN+C60 on $3 \mathrm{~nm}$ PEN. PEN thin film phase peaks are labeled with gray squares, reflections from C60 with triangles, and the black square denotes the (1-10) peak of a PEN bulk phase. Inset: Low angle reflectivity region; $\Theta$ denotes the Bragg angle.

able to impact the structural, surface and electrical properties, which can lead to a device efficiency improvement. Therefore, we investigated the impact of annealing on the PEN + C60 film deposited on the PEN underlayer; the XRD results are shown in Fig. 5(a). From the specular scans it becomes evident that annealing increases the order in the C60 portion, since the as prepared film does not exhibit the second order reflection of C60, which, however, can be observed in all spectra of the annealed samples. Interestingly, the corresponding (002) lattice spacing derived from the (004) reflection is significantly reduced from $0.843 \mathrm{~nm}$ in the sample annealed at $120{ }^{\circ} \mathrm{C}$ to $0.817 \mathrm{~nm}$ for annealing temperatures $\geq 145^{\circ} \mathrm{C}$, which then equals the value found for the pristine $\mathrm{C} 60$ film on PEN. At annealing temperatures higher than $145{ }^{\circ} \mathrm{C}$ no more PEN features are found. Our WHA analysis [Fig. 5(b)] reveals that heating up to $120^{\circ} \mathrm{C}$ did not significantly change the value of $\langle L\rangle_{V}$ determined to $24.5 \pm 1 \mathrm{~nm}$. Moreover no change of the strain value could be observed. Between $120{ }^{\circ} \mathrm{C}$ and $140{ }^{\circ} \mathrm{C}$ the value of $\langle L\rangle_{V}$ is significantly reduced to $14.5 \pm 1 \mathrm{~nm}$, and further to $13.5 \pm 1 \mathrm{~nm}$ for $145{ }^{\circ} \mathrm{C}$ thus indicating desorption of PEN from the film. From these findings we can deduce a PEN desorption temperature higher than $120^{\circ} \mathrm{C}$ (at ambient pressure) hence demonstrating the possibility to make use of postfabrication annealing of $\mathrm{PEN}+\mathrm{C} 60$ heterostructures at this temperature. Note that annealing of the PEN+C60 film on a bare substrate (i.e., without PEN precoverage) up to $150{ }^{\circ} \mathrm{C}$ did not provide evidence for a crystallization of $\mathrm{C} 60$ (not shown).

To investigate the validity of the above findings for the application relevant substrate PEDOT:PSS on ITO coated glass, we performed specular XRD measurements on pure C60 $(\theta=20 \mathrm{~nm})$ and PEN $(\theta=20 \mathrm{~nm})$ films and on a codeposited PEN+C60 (total $\theta=40 \mathrm{~nm}$ ) film on the bare substrate and on a thin PEN underlayer $(\theta=3 \mathrm{~nm})$; the results are shown in Fig. 6. We found evidence for amorphous growth of C60 in the pure and codeposited film on the bare 

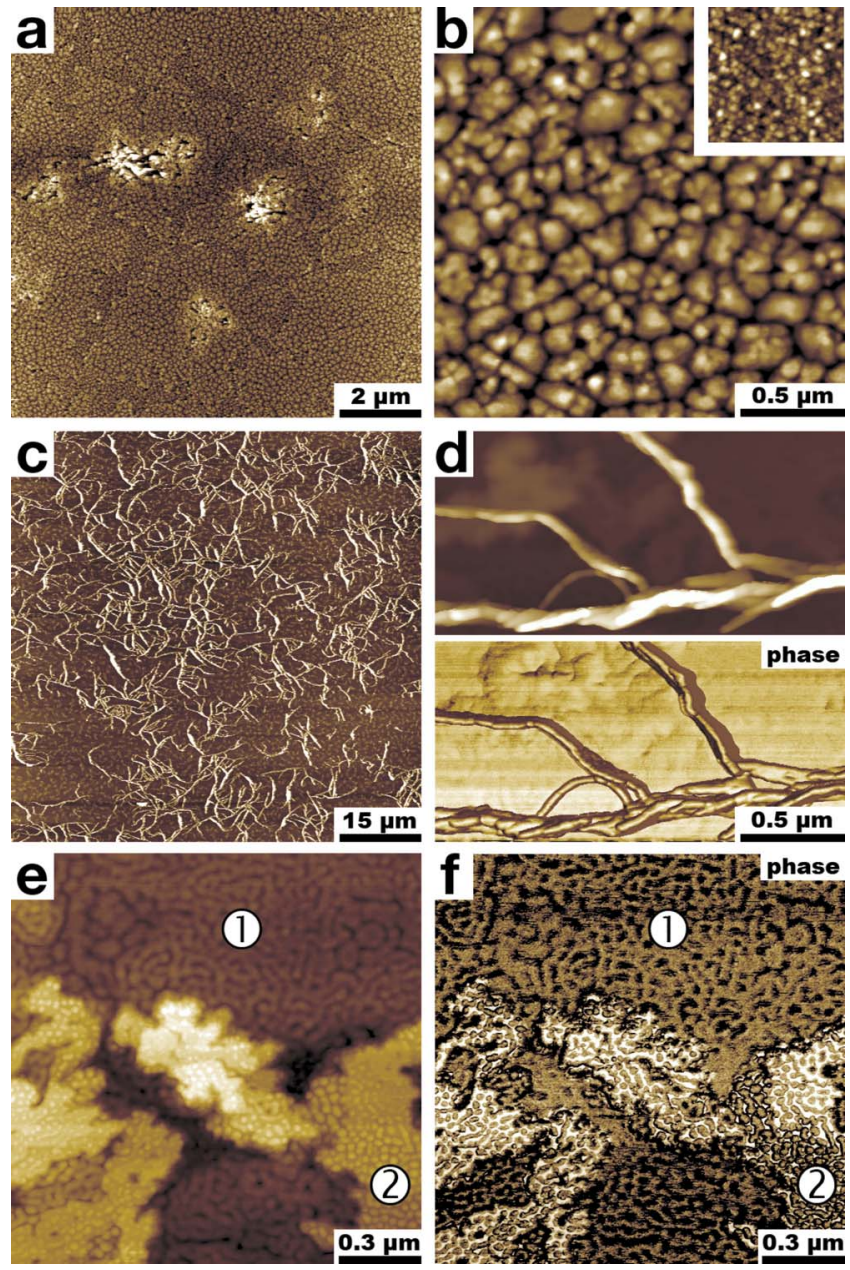

FIG. 7. (Color online) AFM micrographs of nominally $30 \mathrm{~nm}$ C60 on a 3 $\mathrm{nm}$ PEN underlayer [(a) and (b)] and on the bare $\mathrm{SiO}_{2}$ substrate [inset of same scale in (b)] and of $20 \mathrm{~nm} \mathrm{PEN}+\mathrm{C} 60$ on $3 \mathrm{~nm}$ PEN covered $\mathrm{SiO}_{2}$ [(c)-(f)], where (d)-(f) are close-up scans of representative areas of interest. Colors correspond to height levels up to: $2 \mathrm{~nm}$ in (a) and (b), $200 \mathrm{~nm}$ in (c) and (d), and $20 \mathrm{~nm}$ in (e) and (f) and to phase values of up to $90^{\circ}$ in (d) and (f). Areas of interest in the interneedle regions (e)-(f) are labeled with (1) and (2).

PEDOT:PSS substrate as well as (001) textured growth of PEN in the thin film phase. In the case of the PEN precovered substrate, we found the (002) reflection of the hexagonal C60 polymorph in complete analogy to our findings on $\mathrm{SiO}_{2}$. Note that in the low angle region of the spectra two critical angles $\left(\alpha_{c, 1}, \alpha_{c, 2}\right)$ can be observed that we attribute to the PEDOT:PSS layer as well as to the underlying ITO/glass substrate, respectively. This allows to estimate the mean electron density of the PEDOT:PSS layer to $\varrho_{\mathrm{el}, 1}$ $=0.51 e \AA^{-3} \cdot 54,55$

\section{MORPHOLOGY}

We performed additional AFM investigations using topography and phase imaging in order to correlate the results from our structural investigations to morphological properties; representative micrographs are depicted in Fig. 7. A film of $\mathrm{C} 60$ on the PEN precovered $\mathrm{SiO}_{2}$ substrate exhibited a granular morphology (RMS roughness $=3.1 \mathrm{~nm}$ ) comparable to previous reports ${ }^{27,56}$ with an island density of (90 \pm 10$) \mu \mathrm{m}^{-2}$, which was about 30 times lower than for

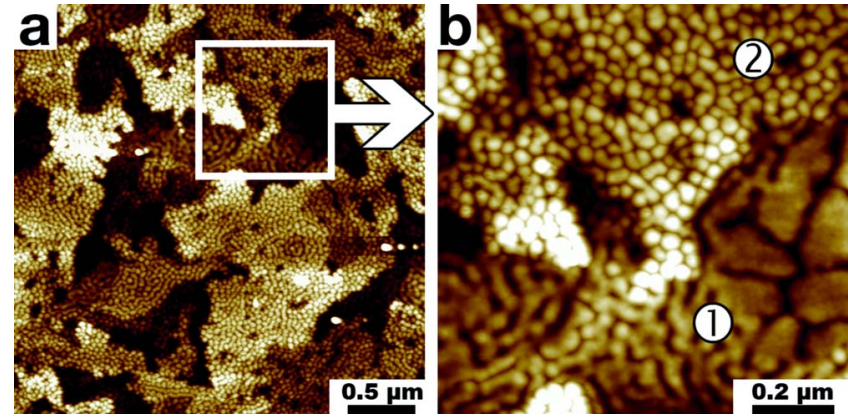

FIG. 8. (Color online) AFM micrograph of nominally $20 \mathrm{~nm}$ thick PEN $+\mathrm{C} 60$ films on a $3 \mathrm{~nm}$ PEN underlayer after annealing at $150{ }^{\circ} \mathrm{C}$ for 120 min (a) and a zoomed view (b); colors correspond to height levels of up to $20 \mathrm{~nm}$.

$\mathrm{C} 60$ on the bare substrate [RMS roughness $=1.6 \mathrm{~nm}$; see inset of Fig. 7(b)]. AFM topography micrographs of the PEN + C60 film on the PEN precovered substrate are shown in Figs. 7(c)-7(e). The most apparent feature is a needle-like network with an elevation of up to $200 \mathrm{~nm}$ exhibiting an internal structure best visible in the corresponding phase image [Fig. 7(d)]. The area between these islands [Fig. 7(e)] exhibits a variety of morphologies: areas of meandered lamellae (1) as well as areas of round islands (2) embedded within a smooth matrix. These morphologies strikingly resemble patterns often found in phase-separated block copolymers and polymer blend films. ${ }^{57-59}$ AFM phase imaging can provide high contrast due to variations in local attractive forces and stiffness and can therefore be used as tool to discriminate between areas covered by different chemical compounds in blended films. ${ }^{60-62}$ The phase image in Fig. 7(f) reveals comparably the same phase angle values $(\varphi)$ in the meandered lamellae (1) and the round islands (2), however, with high contrast to the surrounding smooth matrix in (2) [white areas in Fig. 7(e)] with a phase angle variation of $\Delta \varphi \approx 20^{\circ}$. We interpret this finding as nanophase separation between C60 (islands) and PEN (matrix) in area (2).

The assignment of the morphological features to the distinct materials can be corroborated via AFM investigation of the PEN $+\mathrm{C} 60$ on PEN sample heated to $150^{\circ}$, where the desorption of PEN was confirmed via XRD (see Sec. V). In large area AFM micrographs no more needle-like crystallites like in Figs. 7(c) and 7(d) could be observed, whereas the close-view on the structure of the remaining film in Fig. 8 almost perfectly resembles the morphology of the as prepared film, however, without the smooth matrix surrounding the round islands. In the corresponding phase images $\Delta \varphi$ of neighboring features did not significantly vary as was the case for the as prepared film. These findings are evidence that both the needle-like crystallites with high elevation and the smooth surrounding matrix of the round islands consist of PEN. Moreover, after the annealing process no significant number of pores was observed instead of the PEN matrix, which points to the nanophase-separated PEN portion not penetrating deep into the C60 film and therefore not forming continuous paths to the substrate.

An AFM investigation of the PEN+C60 film ( $\theta$ $=20 \mathrm{~nm})$ on PEN precovered PEDOT:PSS substrates yielded essentially the same results. Again, needle-like struc- 

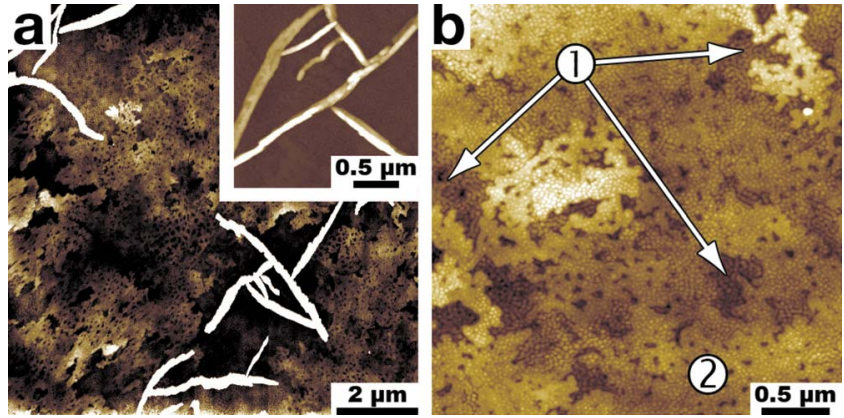

FIG. 9. (Color online) AFM micrographs of a nominally $40 \mathrm{~nm}$ thick PEN+C60 film on a PEDOT:PSS substrate covered with $3 \mathrm{~nm}$ PEN underlayer. Colors correspond to height levels of $30 \mathrm{~nm}$ in (a) and (b) and to 200 $\mathrm{nm}$ [inset in (a)].

tures of up to $200 \mathrm{~nm}$ were found surrounded by a film of low elevation exhibiting again the two different morphologies already found in the film on $\mathrm{SiO}_{2}$; representative micrographs are shown in Fig. 9. However, areas (2) appear to contain more holes on PEDOT:PSS than in the case of growth on $\mathrm{SiO}_{2}$ whereas the island density of the round islands was essentially the same. We attribute these small differences in morphology to the higher surface roughness of PEDOT:PSS (RMS $\approx 1.2 \mathrm{~nm}$ ) compared to $\mathrm{SiO}_{2}$ (RMS $\approx 0.2 \mathrm{~nm}$ ) leading to an increased defect density of the PEN underlayer.

From all investigated samples elevation histograms of the AFM micrographs were derived as shown in Fig. 10. The films of pure $\mathrm{C} 60$ on the bare and the PEN precovered substrate did not exhibit characteristic step heights, whereas all codeposited $\mathrm{PEN}+\mathrm{C} 60$ films on $\mathrm{SiO}_{2}$ showed pronounced overlapping peaks. In the case of the PEN+C60 film on the PEN precovered $\mathrm{SiO}_{2}$ substrate $(\theta=20 \mathrm{~nm})$ we determined the dominating island height $(h)$ to $\approx 11 \mathrm{~nm}$; the same film on the PEDOT:PSS substrate exhibited a slightly lower value of $h \approx 9 \mathrm{~nm}$. Interestingly, the film of twice the nominal film thickness on $\mathrm{SiO}_{2}$ only showed an increase of $h$ to $\approx 13 \mathrm{~nm}$,

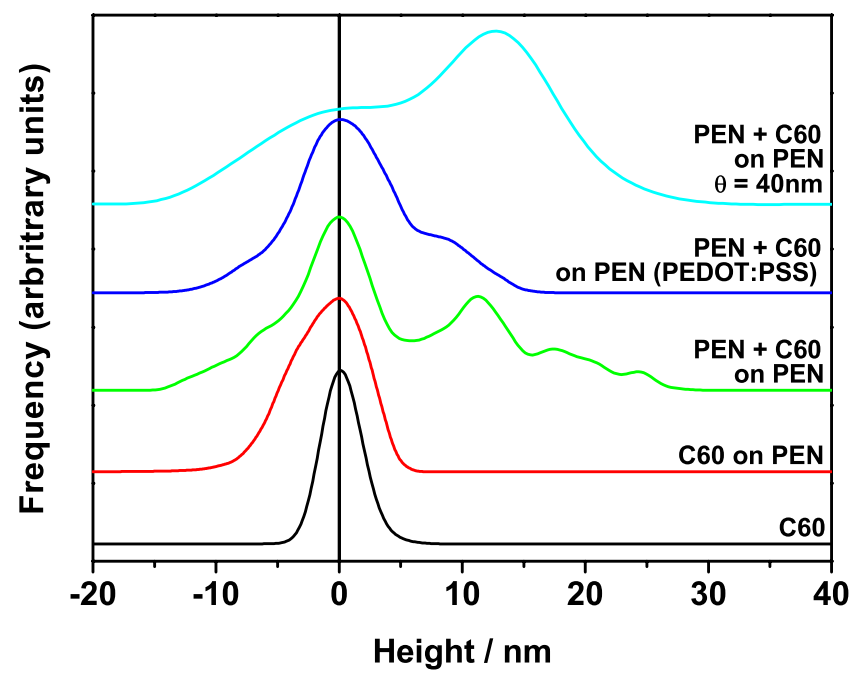

FIG. 10. (Color online) Elevation histograms of AFM micrographs shifted vertically for clarity. If not explicitly stated differently: $\mathrm{SiO}_{2}$ substrates, $\theta$ $=20 \mathrm{~nm}$.

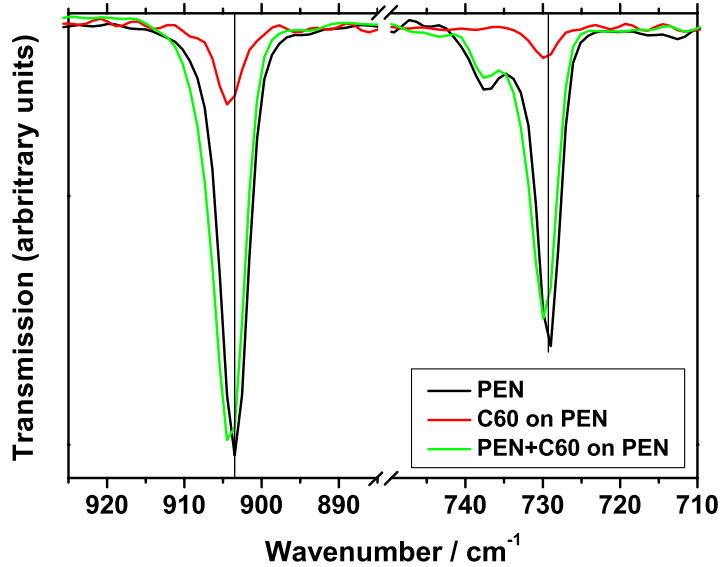

FIG. 11. (Color online) FT-IR results of films of PEN $(\theta=20 \mathrm{~nm})$, C60 $(\theta=30 \mathrm{~nm})$ on $3 \mathrm{~nm}$ PEN and PEN $+\mathrm{C} 60(\theta=40 \mathrm{~nm})$ on $3 \mathrm{~nm}$ PEN.

whereas the frequency ratio to the peak at the origin was reversed thus indicating preferential lateral instead of vertical growth at increasing $\theta$.

\section{VIBRATIONAL SPECTROSCOPY MEASUREMENTS-FT-IR}

From XRD we deduced crystalline growth of PEN in the thin film polymorph for the codeposited films and our AFM investigations revealed that $\mathrm{C} 60$ islands partly grow enclosed within a PEN matrix. Since XRD investigations are exclusively sensitive to crystalline portions of a sample, information on the structural arrangement of possibly amorphous PEN portions is inaccessible via XRD. Therefore we performed a FT-IR investigation on the pure and codeposited films, since FT-IR probes both crystalline and amorphous sample portions and is additionally sensitive to polymorphism. ${ }^{15}$ Two PEN fingerprint ranges are shown in Fig. 11. The vibrations of the PEN thin film reference sample $(\theta=20 \mathrm{~nm})$ at $729.5 \mathrm{~cm}^{-1}, 737.5 \mathrm{~cm}^{-1}$, and $903.5 \mathrm{~cm}^{-1}$ can be assigned to the C-H out-of-plane bending modes. ${ }^{63,64}$ Together with our XRD results we can assign these vibrations to PEN in the thin film phase (additional pronounced vibrations at 833.0, 954.0, 1296.5, and $\left.1344.0 \mathrm{~cm}^{-1}\right)$. The PEN vibrations of the nominally $3 \mathrm{~nm}$ thick $(\approx 2$ monolayers) underlayer for the crystalline $\mathrm{C} 60$ film appear at the same frequencies, except for the two strong $\mathrm{C}-\mathrm{H}$ out-of-plane bending modes in Fig. 11, which are shifted by $+0.5 \mathrm{~cm}^{-1}$ to $730.0 \mathrm{~cm}^{-1}$ and by $+1 \mathrm{~cm}^{-1}$ to $904.5 \mathrm{~cm}^{-1}$ compared to the PEN reference. This indicates a different molecular arrangement of PEN in the ultrathin film. ${ }^{65}$

The most pronounced vibrations assigned to C60 were observed at $1182.5,1429.0$, and $1539.5 \mathrm{~cm}^{-1}$ in all investigated samples; i.e., amorphous and crystalline grown C60 could not be distinguished in FT-IR. In contrast, the two PEN peaks of the codeposited film (Fig. 11) were found shifted compared to the PEN reference at 730.0 and $904.0 \mathrm{~cm}^{-1}$, the full width at half maximum of the two peaks fitted with a Cauchy function was 3.1 and $3.9 \mathrm{~cm}^{-1}$ for the pure PEN film and 3.7 and $4.5 \mathrm{~cm}^{-1}$ for the codeposited film. Since in WHA the crystalline PEN portion in the codeposited film exhibited comparable crystalline quality and the same struc- 
ture as the pure PEN film, this broadening points to a superposition of the infrared absorption feature of the crystalline and amorphous PEN, which cannot be resolved in our FT-IR experiment. This finding supports our interpretation of the AFM micrographs of the codeposited films of round C60 islands being embedded into a matrix of amorphous PEN and crystalline PEN growing in large needle-like islands.

\section{SUMMARY AND DISCUSSION}

Using various complementary experimental techniques, we derived a comprehensive picture of electronic, structural, and morphological properties of PEN and C60 heterostructures. Starting with the experimental finding of OPVCs based on codeposited PEN +C60 films showing poor performance compared to layered structures of C60/PEN we systematically investigated the possible reasons: (i) unfavorable interface energetics, (ii) structural disorder, (iii) lack of phase separation, and (iv) degree of surface corrugation.

(i) Our UPS results (Fig. 3) prove that PEN prepatterning of the PEDOT:PSS substrate leads to an identical HOMO-LUMO level offset between PEN and C60 in the layered case and in the bulk heterojunction. This was explained by vacuum level alignment of C60 on both PEDOT:PSS and PEN, whereas PEN was Fermilevel pinned on PEDOT:PSS leading to a decreased substrate work function for C60. Therefore, PEN precovered substrates allow for a more favorable HOMO-LUMO level offsets for bulk heterojunction based OPVCs, which is crucial for the exciton dissociation process at the PEN-C60 interface. However, the finding of identical HOMO-LUMO level offsets in cases with PEN precoverage cannot explain the exceedingly different OPVC performance (in Fig. 2).

(ii) Structural investigations with XRD (Fig. 4) revealed that PEN precoverage has an ordering effect on C60, which formed amorphous films on the bare substrates ( $\mathrm{SiO}_{2}$ and PEDOT:PSS), and crystalline films on the PEN thin film phase (001) plane with C60 in its hexagonal crystal structure growing with the (001) plane of C60 parallel to the contact. The same was found in the case of codeposition, where PEN grew (almost upright standing) (001) textured in the thin film phase independent of the PEN precoverage and C60 was crystalline on the PEN precovered substrates only. From WHA it became clear that the crystalline PEN portion exhibits a coherence length far larger than the nominal PEN mass thickness, thus indicating pronounced three-dimensional growth. This is an unexpected growth behavior for this specific polymorph, since the PEN thin film phase morphology on amorphous substrates is known to be fairly flat. Via WHA in defined ex situ heating experiments (Fig. 5) a lower limit for the annealing temperature not affecting the PEN microstructure while improving the structural order in the $\mathrm{C} 60$ portion of $120^{\circ} \mathrm{C}$ was determined and we demonstrated that annealing up to $150{ }^{\circ} \mathrm{C}$ leads to desorption of PEN. (iii) We found that the growth behavior of the pure and codeposited films on PEDOT:PSS (Fig. 6) was very similar to that on $\mathrm{SiO}_{2}$. The finding of PEN growth in both the pure and the codeposited films in the same polymorph is strong evidence for phase separation with C60. This is further supported by the fact that C60 itself exhibited crystalline growth in a known polymorph on PEN.

(iv) AFM investigations revealed a needle-like network of crystallites with up to $200 \mathrm{~nm}$ height (Fig. 7), which we assign to PEN due to our XRD finding of the PEN coherence length exceeding the nominal film thickness. This morphology has not been observed before for the PEN thin film phase, which usually grows showing pronounced monomolecular steps of ca. 1.5 $\mathrm{nm}$ height that correspond to the (001) lattice spacing. The remarkably large vertical dimensions of the PEN crystallites may lead to direct contact to the cathode forming a leakage path for holes, which could deteriorate the performance of the bulk-heterojunction based OPVCs. The areas between the PEN needles are covered by two distinguishable morphologies of meandered lamellae and round islands surrounded by a smooth matrix. We found evidence for PEN and C60 nanophase separation through pronounced contrast in the AFM phase images. Our assignment was corroborated by an AFM investigation of the PEN + C60 on PEN sample annealed at $150^{\circ}$ (Fig. 8), in which the PEN features in the XRD scans had vanished: no needle-like crystallites and no smooth PEN matrix surrounding the round islands was then observed, while the morphology of the areas attributed to $\mathrm{C} 60$ was not modified through the annealing process. In contrast to the smooth C60/PEN films, the codeposited films with $\theta=20 \mathrm{~nm}$ exhibited a preferred island height of $\approx 11 \mathrm{~nm}$ deduced from the elevation histograms (Fig. 10); a film of $\theta=40 \mathrm{~nm}$ showed a preferred island height of $\approx 13 \mathrm{~nm}$. Note that this value is in good agreement with the coherence length of the C60 crystallites deduced from the peak breadth analysis in XRD $(17 \pm 2 \mathrm{~nm})$ taking into account that preferred island height values are measured relatively between areas labeled (1) and (2) in the micrographs, which both are expected to grow on doubling the nominal film thickness. The morphology of the application relevant films on PEDOT:PSS (Fig. 9) appeared to be essentially the same compared to the films grown on the model substrate $\mathrm{SiO}_{2}$.

Our FT-IR investigations (Fig. 11) revealed a shift and a broadening of the PEN C-H out-of-plane bending modes of the codeposited film compared to a pure PEN reference, which we explain by a superposition of features from PEN portions with different molecular arrangement. Since the PEN crystalline quality in the PEN+C60 film (Fig. 4) was comparable to the pure PEN film, we explain this shift by a superposition of contributions from the needle-like crystallites (thin film phase) and amorphous PEN portions in nanophase-separated areas. 
(a)

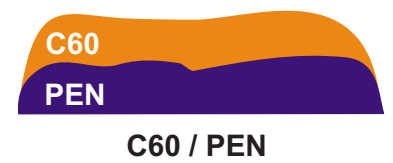

(b)

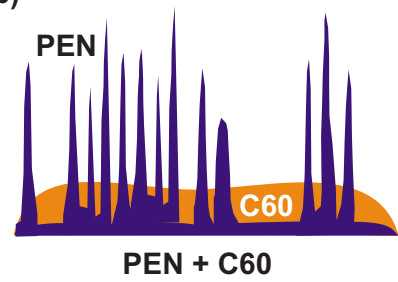

FIG. 12. (Color online) Schematic drawing of growth models deduced from the experimental results for (a) a layered film of C60 on PEN and (b) a codeposited film of PEN+C60 on a thin PEN underlayer.

These findings provide a comprehensive answer to the question of why the OPVC based on the codeposited structure showed poor performance results: (i) The distance between the needle-like PEN islands is in the length scale of micrometer and their height exceeds the surrounding C60 areas by more than one order of magnitude. This minimizes the common interface necessary for an efficient exciton dissociation (cartoon see Fig. 12). (ii) The areas exhibiting nanophase separation most probably do not form continuous PEN paths to the substrate, which is however crucial for the performance of bulk-heterojunction based OPVCs. (iii) The PEN underlayer of $\theta=3 \mathrm{~nm}$ is too thin to significantly contribute to light harvesting and exciton dissociation at the PEN/C60 interface, since exciton quenching occurs in defect-rich ultrathin films.

In this study we demonstrated that a multitechnique view on the physical properties is crucial for a correct interpretation of device performance results. We identified morphological issues to be responsible for poor device performance of PEN + C60 coevaporated OPVCs. Based on our experimental findings a systematic modification of preparation parameters and device structure may be feasible: an increase in the PEN underlayer thickness and a reduction in the PEN island separation distance, e.g., via a significant increase in the deposition rate or via substrate cooling could combine the benefits of the layered and the codeposited structures.

\section{ACKNOWLEDGMENTS}

We thank W. Caliebe (HASYLAB, Hamburg, Germany) for experimental support and Martin Oehzelt and Karin Zojer for fruitful discussions. This work was supported by the Sfb448 and the SPP 1355 (DFG). N.K. acknowledges financial support by the Emmy-Noether-Program (DFG).

${ }^{1}$ C. W. Tang, Appl. Phys. Lett. 48, 183 (1986).

${ }^{2}$ C. W. Tang and S. A. VanSlyke, Appl. Phys. Lett. 51, 913 (1987).

${ }^{3}$ G. Yu, J. Gao, J. C. Hummelen, F. Wudl, and A. J. Heeger, Science 270, 1789 (1995).

${ }^{4}$ N. Koch, ChemPhysChem 8, 1438 (2007)

${ }^{5}$ P. Peumans, S. Uchida, and S. R. Forrest, Nature (London) 425, 158 (2003).

${ }^{6}$ J. Nelson, Science 293, 1059 (2001).

${ }^{7}$ S. Yoo, B. Domercq, and B. Kippelen, Appl. Phys. Lett. 85, 5427 (2004).
${ }^{8}$ A. K. Pandey and J.-M. Nunzi, Appl. Phys. Lett. 89, 213506 (2006).

${ }^{9}$ D. M. Nanditha, M. Dissanayake, A. A. D. T. Adikaari, R. J. Curry, R. A. Hatton, and S. R. P. Silva, Appl. Phys. Lett. 90, 113505 (2007).

${ }^{10}$ P. Sullivan, S. Heutz, S. M. Schultes, and T. S. Jones, Appl. Phys. Lett. 84, 1210 (2004)

${ }^{11}$ B. Brousse, B. Ratier, and A. Moliton, Thin Solid Films 451-452, 81 (2004).

${ }^{12}$ C. J. Brabec, N. S. Sariciftci, and J. C. Hummelen, Adv. Funct. Mater. 11, 15 (2001).

${ }^{13}$ J. Xue, B. P. Rand, S. Uchida, and S. R. Forrest, J. Appl. Phys. 98, 124903 (2005).

${ }^{14}$ J. Xue, S. Uchida, B. P. Rand, and S. R. Forrest, Appl. Phys. Lett. 84, 3013 (2004).

${ }^{15}$ I. Salzmann, R. Opitz, S. Rogaschewski, J. P. Rabe, and N. Koch, Phys. Rev. B 75, 174108 (2007)

${ }^{16}$ I. Salzmann, S. Duhm, R. Opitz, J. P. Rabe, and N. Koch, Appl. Phys. Lett. 91, 051919 (2007).

${ }^{17}$ A. K. Pandey, K. N. N. Unni, and J. Nunzi, Thin Solid Films 511-512, 529 (2006).

${ }^{18}$ P. Peumans, A. Yakimov, and S. R. Forrest, J. Appl. Phys. 93, 3693 (2003b).

${ }^{19}$ H. Ishii, K. Sugiyama, E. Ito, and K. Seki, Adv. Mater. 11, 605 (1999).

${ }^{20}$ A. Kahn, N. Koch, and W. Y. Gao, J. Polym. Sci., Part B: Polym. Phys. 41, 2529 (2003).

${ }^{21}$ N. Koch, I. Salzmann, R. L. Johnson, J. Pflaum, R. Friedlein, and J. P. Rabe, Org. Electron. 7, 537 (2006).

${ }^{22}$ H. Vázquez, W. Gao, F. Flores, and A. Kahn, Phys. Rev. B 71, 041306 (2005).

${ }^{23}$ B. P. Rand, J. Xue, S. Uchida, and S. R. Forrest, J. Appl. Phys. 98, 124902 (2005).

${ }^{24}$ A. H. Jayatissa, T. Gupta, and A. D. Pandya, Carbon 42, 1143 (2004).

${ }^{25}$ N. Koch, A. Vollmer, and A. Elschner, Appl. Phys. Lett. 90, 043512 (2007).

${ }^{26}$ R. L. Johnson and J. Reichardt, Nucl. Instrum. Methods Phys. Res. 208, 791 (1983)

${ }^{27}$ S. Yoo, W. J. Potscavage, Jr., B. Domercq, S. Han, T. Li, S. Jones, R. Szoszkiewicz, D. Levi, E. Riedo, S. R. Marder, and B. Kippelen, SolidState Electron. 51, 1367 (2007).

${ }^{28}$ M. Vogel, S. Doka, C. Breyer, M. C. Lux-Steiner, and K. Fostiropoulos, Appl. Phys. Lett. 89, 163501 (2006).

${ }^{29}$ N. Koch, A. Elschner, J. P. Rabe, and R. L. Johnson, Adv. Mater. 17, 330 (2005).

${ }^{30}$ N. Koch and A. Vollmer, Appl. Phys. Lett. 89, 162107 (2006).

${ }^{31}$ S. Kang, Y. Yi, C. Kim, S. Cho, M. Noh, K. Jeong, and C. Whang, Synth. Met. 156, 32 (2006).

${ }^{32}$ F. J. Zhang, A. Vollmer, J. Zhang, Z. Xu, J. P. Rabe, and N. Koch, Org. Electron. 8, 606 (2007).

${ }^{33}$ Note that the IE of PEN seems remarkably large $(5.50 \mathrm{eV})$ in the codeposited film on pristine PEDOT:PSS. This cannot be explained by a structural reorientation of PEN compared to the pure PEN film (Ref. 66). PEN and C60 exhibit pronounced phase separation (as demonstrated in the XRD and AFM part of this work) with local vacuum levels above the respective patches. In our UPS experiments an area averaged surface potential is measured (Refs. 67-69). Therefore the IE of PEN in the codeposited film cannot be properly determined with the present data.

${ }^{34}$ R. Mitsumoto, K. Seki, T. Araki, E. Ito, Y. Ouchi, Y. Achiba, K. Kikuchi, S. Yajima, S. Kawasaki, F. Okino, H. Touhara, H. Kurosaki, T. Sonoda, and H. Kabayashi, J. Electron Spectrosc. Relat. Phenom. 78, 453 (1996).

${ }^{35}$ The reversed deposition sequence of PEN on C60 precovered PEDOT:PSS is not relevant for OPVC application. However, the results are interesting from a fundamental point of view, since we found evidence for a lack of thermodynamic equilibrium between the PEN top-layer and the substrate, which will be subject of a forthcoming publication (Ref. 70).

${ }^{36}$ C. C. Mattheus, A. B. Dros, J. Baas, G. T. Oostergetel, A. Meetsma, J. L. de Boer, and T. T. M. Palstra, Synth. Met. 138, 475 (2003).

${ }^{37}$ H. Kiessig, Ann. Phys. 402, 715 (1931).

${ }^{38}$ J. L. de Boer, S. van Smaalen, V. Petricek, P. M. Dusek, M. A. Verheijen, and G. Meijer, Chem. Phys. Lett. 219, 469 (1994).

${ }^{39}$ K. Itaka, M. Yamashiro, J. Yamaguchi, M. Haemori, S. Yaginuma, Y. Matsumoto, M. Kondo, and H. Koinuma, Adv. Mater. 18, 1713 (2006).

${ }^{40}$ S. Schiefer, M. Huth, A. Dobrinevski, and B. Nickel, J. Am. Chem. Soc. 129, 10316 (2007).

${ }^{41}$ D. Nabok, P. Puschnig, C. Ambrosch-Draxl, O. Werzer, R. Resel, and D. M. Smilgies, Phys. Rev. B 76, 235322 (2007). 
${ }^{42}$ B. Warren, X-Ray Diffraction (Dover, New York, 1990).

${ }^{43}$ M. Tolan, X-ray Scattering from Soft-Matter Thin Films, Springer Tracts in Modern Physics Vol. 148 (Springer-Verlag, Berlin, 1999).

${ }^{44}$ A. C. Dürr, F. Schreiber, M. Munch, N. Karl, B. Krause, V. Kruppa, and H. Dosch, Appl. Phys. Lett. 81, 2276 (2002).

${ }^{45}$ P. Scherrer, Nachr. Ges. Wiss. Goettingen, Math.-Phys. K1. 2, 98 (1918).

${ }^{46}$ T. Minari, T. Nemoto, and S. Isoda, J. Appl. Phys. 96, 769 (2004).

${ }^{47}$ G. K. Williamson and W. H. Hall, Acta Metall. 1, 22 (1953).

${ }^{48}$ M. Oehzelt, G. Koller, J. Ivanco, S. Berkebile, T. Haber, R. Resel, F. Netzer, and M. Ramsey, Adv. Mater. 18, 2466 (2006).

${ }^{49}$ J. I. Langford, in Accuracy in Powder Diffraction II, National Institute of Standards and Technology Special Publication, (NIST, Washington: US Department of Commerce, 1992) Vol. 846, pp. 110-126.

${ }^{50}$ R. Snyder, J. Fiala, and H. J. Bunge, Defect and Microstructure Analysis by Diffraction (Oxford University Press, New York, 1999).

${ }^{51}$ M. Birkholz, Thin Film Analysis by X-Ray Scattering (Wiley-VCH Verlag $\mathrm{GmbH}$, Weinheim, 2006)

${ }^{52}$ The parameters $\beta_{2 \Theta}$ and $d$ in reciprocal units: $\beta_{2 \Theta}^{*}=\beta_{2 \Theta} \cos (\theta) / \lambda, d^{*}$ $=2 \sin (\theta) / \lambda$.

${ }^{53}$ U. Zhokhavets, T. Erb, H. Hoppe, G. Gobsch, and N. S. Sariciftci, Thin Solid Films 496, 679 (2006).

${ }^{54}$ V. Holý, U. Pietsch, and T. Baumbach, High-Resolution X-Ray Scattering from Thin Films and Multilayers, Springer Tracts in Modern Physics Vol. 149 (Springer-Verlag, Berlin, 1999).

${ }^{55} \varrho_{\mathrm{el}}=\alpha_{c}^{2} \pi / \lambda^{2} r_{\mathrm{el}}$ where $\alpha_{c}$ denotes the Bragg-angle of total external reflection, $\lambda$ the x-ray wavelength, and $r_{\mathrm{el}}=e^{2} / 4 \pi \varepsilon_{0} m_{\mathrm{el}} c^{2} \approx 2.82 \times 10^{-15} \mathrm{~m}$ the Compton radius.

${ }^{56}$ J. Yang and T. Q. Nguyen, Org. Electron. 8, 566 (2007).

${ }^{57}$ I. I. Potemkin and M. Moller, Macromolecules 38, 2999 (2005).

${ }^{58}$ S. Walheim, M. Ramstein, and U. Steiner, Langmuir 15, 4828 (1999).

${ }^{59}$ P. Müller-Buschbaum, E. Bauer, O. Wunnicke, and M. Stamm, J. Phys.: Condens. Matter 17, S363 (2005)

${ }^{60}$ Y. Thomann, H.-J. Cantow, G. Bar, and M.-H. Whangbo, Appl. Phys. A 66, S1233 (1998)

${ }^{61}$ S. N. Magonov, V. Elings, and V. S. Papkov, Polymer 38, 297 (1997).
${ }^{62}$ P. Busch, D. Posselt, D.-M. Smilgies, B. Rheinlander, F. Kremer, and C. Papdakis, Macromolecules 36, 8717 (2003).

${ }^{63}$ J. Szczepanski, C. Wehlburg, and M. Vala, Chem. Phys. Lett. 232, 221 (1995).

${ }^{64}$ D. M. Hudgins and S. A. Sandford, J. Phys. Chem. A 102, 344 (1998).

${ }^{65}$ In a recent XRD study almost upright molecular arrangement was shown for the PEN monolayer ${ }^{71}$ and in a different work it has been reported that PEN exhibits a thickness driven orthorhombic to triclinic phase transition on amorphous carbon coated mica substrates (Ref. 72). The unit cells proposed in both studies are equal within the error margin, hence we may speculate that the vibrations found in our FT-IR investigation can be assigned to this specific polymorph. Another explanation can be the bimodal growth behavior of PEN films in thin film and bulk phase, which was recently shown to be undetectable by XRD at low nominal film thickness (Ref. 73). Therefore the shifted vibration of the ultrathin film could dominantly stem from the thin film phase, whereas the thick PEN film shows a superposition of thin film and bulk phase features. Admittedly, this cannot be decided from our data and more work concerning this question in particular and the correlation of FT-IR to XRD in general is necessary and will be subject to our further research efforts.

${ }^{66}$ S. Duhm, G. Heimel, I. Salzmann, H. Glowatzki, R. L. Johnson, A. Vollmer, J. P. Rabe, and N. Koch, Nature Mater. 7, 326 (2008).

${ }^{67}$ G. Koller, F. P. Netzer, and M. G. Ramsey, Appl. Phys. Lett. 83, 563 (2003).

${ }^{68}$ R. Fischer, S. Schuppler, N. Fischer, T. Fauster, and W. Steinmann, Phys. Rev. Lett. 70, 654 (1993).

${ }^{69}$ I. Salzmann, S. Duhm, G. Heimel, M. Oehzelt, R. Kniprath, R. L. Johnson, J. P. Rabe, and N. Koch, J. Am. Chem. Soc.. 130, 12870 (2008).

${ }^{70}$ S. Duhm, I. Salzmann, R. L. Johnson, and N. Koch (unpublished).

${ }^{71}$ S. E. Fritz, S. M. Martin, C. D. Frisbie, M. D. Ward, and M. F. Toney, J. Am. Chem. Soc. 126, 4084 (2004).

${ }^{72}$ L. Drummy and D. Martin, Adv. Mater. 17, 903 (2005).

${ }^{73}$ A. C. Mayer, A. Kazimirov, and G. G. Malliaras, Phys. Rev. Lett. 97, 105503 (2006) 